\title{
NGHIÊN CỬU XÂY DỰNG PHẦN MỀM TÍNH TOÁN CHİ SỐ CHẤT LƯợNG NƯỚC WQI CHO LU'U VỰC SÔNG SEREPOK
}

\author{
Huỳnh Phú ${ }^{1}$
}

Tóm tắt: Chi số chất lượng nước Water Quality Index (WQI) là một chi số được tính toán tì̀ các thông số quan trắc chất luợng nuớc, dùng để mô tả định lương về chất lượng nước và khả năng sủ dung của nguồn nuớc đó được biểu diễn qua một thang điểm. Hiện nay có rất nhiều quốc gia/ địa phưong xây dưng và áp dụng chỉ số WQI. Thông qua một mô hình tính toán, tù các thông số khác nhau pH, DO, $\mathrm{BOD}_{5}, \mathrm{COD}, \mathrm{NH}_{4}^{+}, \mathrm{PO}_{4}^{2-}$, Coliform...ta thu được một chi số duy nhất. Sau đó chất lương nước có thể được so sánh với nhau thông qua chi số WQI. Theo Quyết định 879/QĐ-TCMT, ngày 01 tháng 7 năm 2011 của Tổng cục Môi trường. Bài báo trình bày, bằng công cu tin học tác giả đã nghiên cưu và xây dựng phần mềm WQI_Serepok nhằm tính toán nhanh chỉ số chất luợng nước WQI, góp phần vào công tác quản lý chất luợng nước sông Serepok.

Từ khóa: Chất lượng nước, WQI, Chịu tải, Sông Serepok, phần mềm WQI_Serepok.

Ban Biên tập nhận bài: 15/04/2019 Ngày phản biện xong: 20/5/2019 Ngày đăng bài: 25/06/2019

\section{1. Đặt vấn đề}

Từ khi "Sổ tay hướng dẫn tính toán chỉ số chất lượng nước" được ra đời theo Quyết định 879/QĐ-TCMT, ngày 01 tháng 7 năm 2011 của Tổng cục Môi trường thông qua chỉ số chất lượng nước (WQI) thì công tác đánh giá chất lượng nước được thực hiện một cách dễ dàng, các kết quả đánh giá phần nào đã đáp ứng được bức tranh về hiện trạng chất lượng nước cho toàn lưu vực sông thông qua các vị trí quan trắc. Trong Quyết định đã trình bày rất cụ thể cách thức thực hiện tính toán chỉ số WQI, bằng phương pháp tính toán thống kê.

Hiện nay, khi chất lượng tài nguyên nước mặt ở nhiều lưu vực sông đang trong tình trạng bị suy thoái, ảnh hưởng rất lớn đến việc khai thác, sử dụng tài nguyên nước. Cũng chính lý do đó mà đã có rất nhiều các dự án, các nghiên cứu đánh giá chất lượng nước nhằm đề xuất các giải pháp quản lý, khai thác hợp lý nguồn tài nguyên nước cho mỗi lưu vực sông. Việc đánh giá hiện trạng chất lượng nước thường được thực hiện bằng phương pháp thống kê dựa theo số liệu quan trắc hàng năm, hoặc số liệu qua những đợt khảo sát thực địa. Mục đích nghiên cứu sử dụng chỉ số WQI nhằm: (1) Đánh giá nhanh chất lượng nước mặt lục địa một cách tổng quát; (2) Có thể được sử dụng như một nguồn dữ liệu để xây dựng bản đồ phân vùng chất lượng nước; (3) Cung cấp thông tin môi trường cho cộng đồng một cách đơn giản, dễ hiểu, trực quan; (4) Nâng cao nhận thức về môi trường.

\section{Phương án nghiên cứu}

\subsection{Giới thiệu về lưu vục nghiên cúu}

Lưu vực Serepok bao gồm: Phần lớn diện tích tỉnh Đắk Lắk $\left(10.400 \mathrm{~km}^{2}\right)$, một phần diện tích tỉnh Đắk Nông $\left(3.600 \mathrm{~km}^{2}\right)$, một phần diện tích tỉnh Gia Lai $\left(2.900 \mathrm{~km}^{2}\right)$ Một phần nhỏ diện tích tỉnh Lâm Đồng $\left(1.300 \mathrm{~km}^{2}\right)$. Tổng diện tích lưu vực trong lãnh thổ Việt Nam là 18.264 km².

${ }^{1}$ Trưòng Đại học công nghệ TP Hồ Chí Minh

Email:h.phu@hutech.edu.vn 


\section{BÀI BÁO KHOA HỌC}

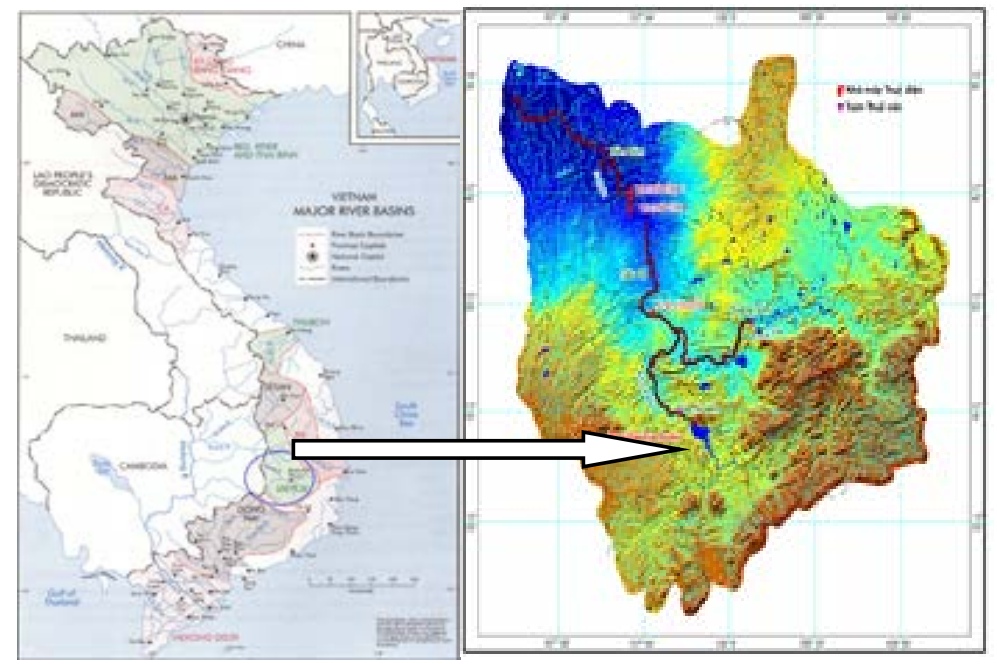

Hình 1. Luu vực sông Sêrêpok

\subsection{Thu thập và xử lý số liệu}

Số liệu thu thập được là kết quả quan trắc của Trung tâm quan trắc môi trường, đề tài nghiên cứu với Phòng Tài nguyên nước Sở Tài nguyên môi trường Đắk lắk, qua các năm 2016, 2017, 2018, 2019. Chọn lọc các thông số quan trọng từ các tài liệu của tỉnh. Xử lý và phân tích các số liệu thu thập được.

\subsection{Phương pháp khảo sát}

Tiến hành khảo sát thực tế tại vị trí địa bàn nghiên cứu, tập trung vào các vị trí tập trung khu dân cư, khu công nghệp, nơi có lưu lượng xả thải lớn trên lưu vực sông.

\subsection{Phương pháp tính toán WQI}

a. Tính toán WQI thông số

WQI thông số (WQISI) được tính toán cho các thông số $\mathrm{BOD}_{5}, \mathrm{COD}, \mathrm{N}-\mathrm{NH}_{4}, \mathrm{P}^{-} \mathrm{PO}_{4}$, TSS, độ đục, Tổng Coliform theo công thức như sau:

$$
\mathrm{WQI}_{\mathrm{SI}}=\frac{\mathrm{q}_{\mathrm{i}}-\mathrm{q}_{\mathrm{i}+1}}{\mathrm{BP}_{\mathrm{i}+1}-\mathrm{BP}_{\mathrm{i}}}\left(\mathrm{BP}_{\mathrm{i}+1}-\mathrm{C}_{\mathrm{p}}\right)+\mathrm{q}_{\mathrm{i}+1}
$$

Trong đó $\mathrm{BP}_{\mathrm{i}}$ là nồng độ giới hạn dưới của giá trị thông số quan trắc được quy định trong bảng 1 tương ứng với mức $\mathrm{I} ; \mathrm{BP}_{\mathrm{i}+1}$ là Nồng độ giới hạn trên của giá trị thông số quan trắc được quy định trong bảng 1 tương ứng với mức $\mathrm{i}+1 ; \mathrm{q}_{\mathrm{i}}$ là giá trị WQI ở mức $\mathrm{i}$ đã cho trong bảng tương ứng với giá trị $\mathrm{BP}_{\mathrm{i}} ; \mathrm{q}_{\mathrm{i}+1}$ là giá trị $\mathrm{WQI}$ ở mức $\mathrm{i}+1$ cho trong bảng tương ứng với giá trị $\mathrm{BP}_{\mathrm{i}+1} ; \mathrm{C}_{\mathrm{p}}$ là giá trị của thông số quan trắc được đưa vào tính toán.

Bảng 1. Bảng quy định các giá trị $q_{i}, B P_{i}$

\begin{tabular}{ccccccccc}
\hline & & \multicolumn{7}{c}{ Giá trị BPi quy định đối với từng thông số } \\
\cline { 3 - 8 } $\mathrm{i}$ & $\mathrm{q}_{\mathrm{i}}$ & $\begin{array}{c}\mathrm{BOD} 5 \\
(\mathrm{mg} / \mathrm{l})\end{array}$ & $\begin{array}{c}\mathrm{COD} \\
(\mathrm{mg} / \mathrm{l})\end{array}$ & $\begin{array}{c}\mathrm{N}-\mathrm{NH}_{4} \\
(\mathrm{mg} / \mathrm{l})\end{array}$ & $\begin{array}{c}\mathrm{P}^{\mathrm{PO}} 4 \\
(\mathrm{mg} / \mathrm{l})\end{array}$ & $\begin{array}{c}\text { Độ đục } \\
(\mathrm{NTU})\end{array}$ & $\begin{array}{c}\mathrm{TSS} \\
(\mathrm{mg} / \mathrm{l})\end{array}$ & $\begin{array}{c}\text { Coliform } \\
(\mathrm{MPN} / 100 \mathrm{ml})\end{array}$ \\
\hline 1 & 100 & $\leq 4$ & $\leq 10$ & $\leq 0.1$ & $\leq 0.1$ & $\leq 5$ & $\leq 20$ & $\leq 2500$ \\
2 & 75 & 6 & 15 & 0.2 & 0.2 & 20 & 30 & 5000 \\
3 & 50 & 15 & 30 & 0.5 & 0.3 & 30 & 50 & 7500 \\
4 & 25 & 25 & 50 & 1 & 0.5 & 70 & 100 & 10.000 \\
5 & 1 & $\geq 50$ & $\geq 80$ & $\geq 5$ & $\geq 6$ & $\geq 100$ & $>100$ & $>10.000$ \\
\hline
\end{tabular}

Ghi chú: Truờng hợp giá trị $C_{p}$ của thông số trùng với giá trị $B P_{i}$ đã cho trong bảng, thì xác định được WQI của thông số chính bằng giá trị qi tương ứng. 
* Tính giá trị WQI đối với thông số DO (WQIDO) được tính toán thông qua giá trị DO $\%$ bão hòa.

Bước 1: Tính toán giá trị DO \% bão hòa:

- Tính giá trị DO bão hòa:

$\mathrm{DO}_{\text {bão hòa }}=14.652-0,41022 \mathrm{~T}+0,0079910$ $\mathrm{T}^{2}-0,000077774 \mathrm{~T}^{3}$

Trong đó $\mathrm{T}$ là nhiệt độ môi trường nước tại thời điểm quan trắc $\left({ }^{\circ} \mathrm{C}\right)$.

- Tính giá trị DO \% bão hòa:
$\mathrm{DO} \%$ bão hòa $=\mathrm{DO}_{\text {hòa tan }} / \mathrm{DO}_{\text {bão hòa }} * 100$

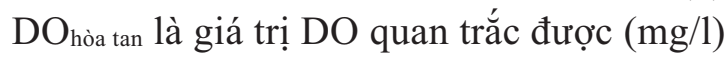

Bước 2: Tính giá trị WQIDO:

$$
W_{\text {QI }}=\frac{q_{i+1}-q_{i}}{{B P_{i+1}}-B P_{i}}\left(C_{p}-B P_{i}\right)+q_{i}
$$

Trong đó $\mathrm{C}_{\mathrm{p}}$ là giá trị $\mathrm{DO} \%$ bão hòa; $\mathrm{BP}_{\mathrm{i}}$, $\mathrm{BP}_{\mathrm{i}+1}, \mathrm{q}_{\mathrm{i}}, \mathrm{q}_{\mathrm{i}+1}$ là các giá trị tương ứng với mức $\mathrm{i}$, i+1 trong Bảng 2.

Bảng 2. Bảng quy định các giá trị $B P_{i}$ và q $q_{i}$ đối với $D O \%$ bão hòa

\begin{tabular}{ccccccccccc}
\hline $\mathrm{i}$ & 1 & 2 & 3 & 4 & 5 & 6 & 7 & 8 & 9 & 10 \\
\hline $\mathrm{BP}_{\mathrm{i}}$ & $\leq 20$ & 20 & 50 & 75 & 88 & 112 & 125 & 150 & 200 & $\geq 200$ \\
$\mathrm{q}_{\mathrm{i}}$ & 1 & 25 & 50 & 75 & 100 & 100 & 75 & 50 & 25 & 1 \\
\hline
\end{tabular}

Nếu giá trị $\mathrm{DO} \%$ bão hòa $\leq 20$ thì WQI 1 ; Nếu $20<$ giá trị $\mathrm{DO} \%$ bão hòa $<88$ thì WQIDO được tính theo công thức 2 và sử dụng Bảng 2; Nếu $88 \leq$ giá trị $\mathrm{DO} \%$ bão hòa $\leq 112$ thì WQIDO bằng 100 ; Nếu $112<$ giá trị $\mathrm{DO} \%$ bão hòa $<200$ thì $\mathrm{WQI}_{\mathrm{DO}}$ được tính theo công thức 1 và sử dụng Bảng 2; Nếu giá trị $\mathrm{DO} \%$ bão hòa $\geq 200$ thì $\mathrm{WQI}_{\mathrm{DO}}$ bằng 1 .

* Tính giá trị WQI đối với thông số $\mathrm{pH}$ Bảng 3. Bảng quy định các giá trị $B P_{i}$ và q $q_{i}$ đối với thông số $p H$

\begin{tabular}{ccccccc}
\hline $\mathrm{I}$ & 1 & 2 & 3 & 4 & 5 & 6 \\
\hline $\mathrm{BP}_{\mathrm{i}}$ & $\leq 5.5$ & 5.5 & 6 & 8.5 & 9 & $\geq 9$ \\
$\mathrm{q}_{\mathrm{i}}$ & 1 & 50 & 100 & 100 & 50 & 1 \\
\hline
\end{tabular}

Nếu giá trị $\mathrm{pH} \leq 5,5$ thì $\mathrm{WQI}_{\mathrm{pH}}$ bằng 1 ; Nếu $5,5<$ giá trị $\mathrm{pH}<6$ thì $\mathrm{WQI}_{\mathrm{pH}}$ được tính theo công thức 2 và sử dụng bảng 3 ; Nếu $6 \leq \mathrm{pH} \leq$ 8,5 thì $\mathrm{WQI}_{\mathrm{pH}}$ bằng 100 ; Nếu $8,5<\mathrm{pH}<9$ thì $\mathrm{WQI}_{\mathrm{pH}}$ được tính theo công thức 1 và sử dụng bảng 3 ; Nếu giá trị $\mathrm{pH} \geq 9$ thì $\mathrm{WQI}_{\mathrm{pH}}$ bằng 1 .

b. Tính toán WQI

Sau khi tính toán WQI đối với từng thông số nêu trên, việc tính toán WQI được áp dụng theo công thức sau:

$$
\mathrm{WQI}=\frac{\mathrm{WQI}_{\mathrm{pH}}}{100}\left[\frac{1}{5} \sum_{\mathrm{a}=1}^{5} \mathrm{WQI}_{\mathrm{a}} \times \frac{1}{2} \sum_{\mathrm{b}=1}^{2} \mathrm{WQI}_{\mathrm{b}} \times \mathrm{WQI}_{\phi}\right]^{1 / 3}
$$

Trong đó WQIa là giá trị WQI đã tính toán đối với 05 thông số: $\mathrm{DO}, \mathrm{BOD}_{5}, \mathrm{COD}, \mathrm{N}^{-\mathrm{NH}_{4}}$, $\mathrm{P}_{-} \mathrm{PO}_{4}$; $\mathrm{WQI}_{\mathrm{b}}$ là giá trị WQI đã tính toán đối với 02 thông số: TSS, độ đục; WQI c là giá trị WQI đã tính toán đối với thông số Tổng Coliform; $\mathrm{WQI}_{\mathrm{pH}}$ là giá trị WQI đã tính toán đối với thông số pH (Giá trị WQI sau khi tính toán sẽ được làm tròn thành số nguyên).

\subsection{Phuơng pháp tư động hóa tin học}

Xây dựng phần mềm WQI_Srepok bằng ngôn ngữ Visual Basic 6.0, sử dụng cho mọi loại hệ điều hành Widowns XP, 7, 8 hoặc $10 \ldots$

\subsection{Phương pháp so sánh, đánh giá}

So sánh chỉ số chất lượng nước đã được tính toán với bảng đánh giá. Sau khi tính toán được WQI, sử dụng bảng xác định giá trị WQI tương ứng với mức đánh giá chất lượng nước để so sánh, đánh giá, cụ thể như sau: 
Bảng 4. So sánh chỉ số chất lương nuớc đã được tính toán với bảng đánh giá

\begin{tabular}{|c|c|c|}
\hline Giá trị WQI & Mức đánh giá chất lượng nước & Màu \\
\hline $91-100$ & Sử dụng tốt cho mục đích cấp nước sinh hoạt & Xanh nước biển \\
\hline $76-90$ & $\begin{array}{l}\text { Sử dụng cho mục đích cấp nước sinh hoạt nhưng cần } \\
\text { các biện pháp xử lý phù hợp }\end{array}$ & Xanh lá cây \\
\hline $51-75$ & $\begin{array}{l}\text { Sử dụng cho mục đích tưới tiêu và các mục đích tương } \\
\text { đương khác }\end{array}$ & Vàng \\
\hline $26-50$ & $\begin{array}{l}\text { Sử dụng cho giao thông thủy và các mục đích tương } \\
\text { đương khác }\end{array}$ & Da cam \\
\hline $0-25$ & $\begin{array}{l}\text { Nước ô nhiễm nặng, cần các biện pháp xử lý trong } \\
\text { tương lai }\end{array}$ & Đỏ \\
\hline
\end{tabular}

\section{Kết quả nghiên cứu}

\subsection{Phần mềm tin học hóa WQI_Serepok}

Phần mềm hiển "WQI_Srepok" được xây dựng bằng ngôn ngữ Visual Basic 6.0, do vậy nó có tính tương thích cao, sử dụng cho mọi loại hệ điều hành: WINDOWS XP, 7, 8 hoặc $10 \ldots$.

Giao diện của phần mềm được thể hiện trên hình 2 . Hệ thống khối điều hành của phần mềm bao gồm:

- Khối “Chọn chỉ tiêu”: Đây là phần lựa chọn các chỉ tiêu để tính toán chỉ số

WQI. Theo số liệu điều tra, có số liệu của chỉ tiêt nào "tích" vào chỉ tiêu đó;

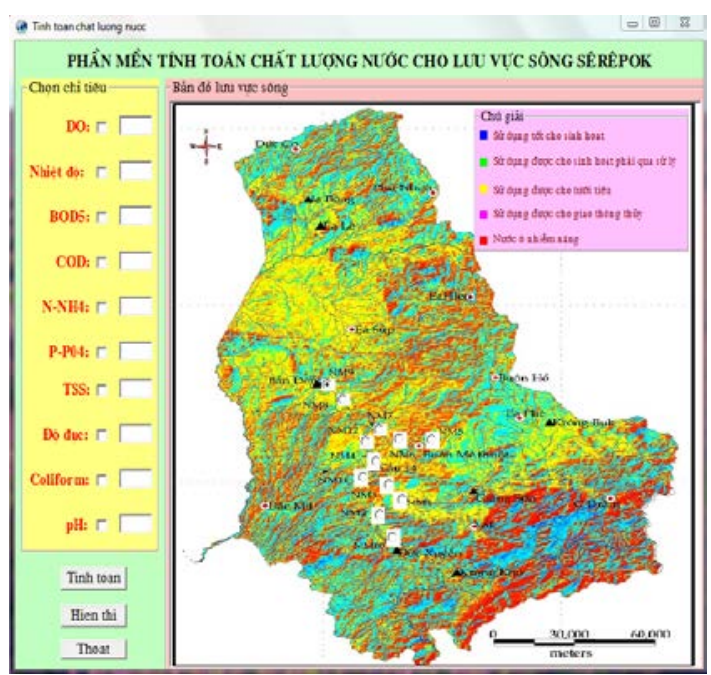

Hình 2. Giao diện của phần mềm WQI_Serepok

- Khối "Bản đồ lưu vực sông”: Đây là vùng lựa chọn các vị trí quan trắc chất lượng nước trên lưu vực sông Serepok, đồng thời cũng là vùng hiển thị kết quả Chât lượng nước theo các cấp độ khác nhau;

- Khối "Nút lệnh thực hiện" bao gồm:

+ Nút "Tinh toán": Lấy dữ liệu và tính toán chỉ số WQI.

+ Nút "Hiển thị": Hiển thị kết quả trên Chất lượng nước bản đồ.

+ Nút "Thoát": Tắt chương trình

\subsection{Code của phần mềm}

Private Sub Tinhtoan_Click()

Dim Out As String, ExaT1 As String, ExaT2 As String, ExaT3 As String, FInput As String

Dim BOD(5) As Variant, COD(5) As Variant, NH4(5) As Variant, PO4(5) As Variant

Dim WQ1(5) As Variant, NTU(5) As Variant, TSS(5) As Variant, CLF(5) As Variant

Dim WQ2(10) As Variant, WDO(10) As Variant, WQ3(6) As Variant, pH(6) As Variant

Dim WQIBOD As Variant, WQICOD As Variant, WQINH4 As Variant, WQIPO4 As Variant

Dim WQINTU As Variant, WQITSS As Variant, WQICLF As Variant, Txt As Variant

Dim DOBH As Variant, DOPBH As Variant, WQIDO As Variant, WQIpH As Variant

Dim WQI As Variant, WQI1 As Variant, WQI2 As Variant, dem As Variant

Dim Tx1 As Variant, Tx2 As Variant, Tx3 As 
Variant, Tx4 As Variant, Tx5 As Variant

Dim Tx6 As Variant, Tx7 As Variant, Tx8 As

Variant, Tx9 As Variant, Tx10 As Variant

Out $=$ App.Path \& "\WQI.DXT"

ExaT1 $=$ App.Path \& "\ExaT1.DXT"

ExaT2 $=$ App.Path \& "\ExaT2.DXT"

ExaT3 $=$ App.Path \& "\ExaT3.DXT"

FInput $=$ App.Path \& "\CLN.txt"

Open ExaT1 For Input As 1

For $\mathrm{i}=1$ To 5

Input \#1, WQ1(i), BOD(i), COD(i), NH4(i),

PO4(i), NTU(i), TSS(i), CLF(i)

Next

Close (1)

Open ExaT2 For Input As 1

Input \#1, $\operatorname{WDO}(1), \operatorname{WDO}(2), \operatorname{WDO}(3)$, WDO(4), $\operatorname{WDO}(5), \quad \operatorname{WDO}(6), \quad \operatorname{WDO}(7)$, $\mathrm{WDO}(8), \mathrm{WDO}(9), \mathrm{WDO}(10)$

Input \#1, WQ2(1), WQ2(2), WQ2(3), WQ2(4), WQ2(5), WQ2(6), WQ2(7), WQ2(8), WQ2(9), WQ2(10)

Close (1)

Open ExaT3 For Input As 1

Input \#1, pH(1), pH(2), pH(3), $\mathrm{pH}(4), \mathrm{pH}(5)$, $\mathrm{pH}(6)$

Input \#1, WQ3(1), WQ3(2), WQ3(3), WQ3(4), WQ3(5), WQ3(6)

Close (1)

Open FInput For Input As 1

'Flat pH TSS DODUC DO BOD5 COD NH4 PO43 Coliforms $\mathrm{T}$

Input \#1, Tx1, Tx2, Tx3, Tx4, Tx5, Tx6, Tx7, Tx8, Tx9, Tx10

Close (1)

If Check1.Value Then

Text1.Text $=\mathrm{Tx} 4$

Text2.Text $=\mathrm{Tx} 10$

DOBH $=14.652-0.41022 *$ Text2.Text + $0.007991 *$ Text2.Text * Text2.Text $0.000077774 *$ Text2.Text $*$ Text2.Text $*$ Text2.Text

DOPBH $=\operatorname{Val}($ Text1.Text $) * 100 / \mathrm{DOBH}$

If $(\operatorname{Val}(\mathrm{DOPBH})<\operatorname{Val}(\mathrm{WDO}(1)))$ Then

$\mathrm{WQIDO}=1$

ElseIf $(\operatorname{Val}($ DOPBH) $>=\operatorname{Val}(\operatorname{WDO}(10)))$
Then

$$
\mathrm{WQIDO}=1
$$

Else

For $\mathrm{i}=1$ To 9

If $((\mathrm{Val}(\mathrm{DOPBH})>=\mathrm{Val}(\mathrm{WDO}(\mathrm{i})))$ And $(\operatorname{Val}(\mathrm{DOPBH})<\operatorname{Val}(\mathrm{WDO}(\mathrm{i}+1))))$ Then

$\mathrm{WQIDO}=((\mathrm{WQ2}(\mathrm{i}+1)-\mathrm{WQ} 2(\mathrm{i})) *$

$(\mathrm{DOPBH}-\mathrm{WDO}(\mathrm{i})) /(\mathrm{WDO}(\mathrm{i}+1)-\mathrm{WDO}(\mathrm{i})))$

+ WQ2 $(i+1)$

End If

Next

End If

Else

$\mathrm{WQIDO}=0$

End If

If Check3.Value Then

Text3.Text $=\mathrm{Tx} 5$

If $(\operatorname{Val}($ Text3.Text $)<\operatorname{Val}(B O D(1)))$ Then

$\mathrm{WQIBOD}=100$

ElseIf $(\operatorname{Val}($ Text3.Text $)>=\operatorname{Val}(\operatorname{BOD}(5)))$ Then

WQIBOD $=1$

Else

For $\mathrm{i}=1$ To 4

If $((\operatorname{Val}($ Text3.Text $)>=\operatorname{Val}(\mathrm{BOD}(\mathrm{i})))$ And $(\operatorname{Val}($ Text3.Text $)<\operatorname{Val}(B O D(i+1))))$ Then

WQIBOD $=(($ WQ1 $(\mathrm{i})-\mathrm{WQ} 1(\mathrm{i}+1)) *$ $(\operatorname{BOD}(\mathrm{i}+1)-$ Text3.Text $) /(\operatorname{BOD}(\mathrm{i}+1)-$ BOD(i))) + WQ1(i+1)

End If

Next

End If

Else

$\mathrm{WQIBOD}=0$

End If

If Check4.Value Then

Text4. Text $=$ Tx 6

If $(\operatorname{Val}($ Text4.Text $)<\operatorname{Val}(\operatorname{COD}(1)))$ Then

$\mathrm{WQICOD}=100$

ElseIf $(\operatorname{Val}($ Text4.Text $)>=\operatorname{Val}(\operatorname{COD}(5)))$ Then

WQICOD $=1$

Else

For $\mathrm{i}=1$ To 4

If $((\operatorname{Val}($ Text4.Text $)>=\operatorname{Val}(\operatorname{COD}(\mathrm{i})))$ And 
$(\operatorname{Val}($ Text4.Text $)<\operatorname{Val}(\operatorname{COD}(i+1))))$ Then

WQICOD $=(($ WQ1 $(\mathrm{i})-\mathrm{WQ} 1(\mathrm{i}+1)) *$ $(\operatorname{COD}(\mathrm{i}+1)-$ Text4.Text $) /(\operatorname{COD}(\mathrm{i}+1)-$ COD(i))) + WQ1(i + 1)

End If

Next

End If

Else

$\mathrm{WQICOD}=0$

End If

If Check5.Value Then

Text5.Text $=$ Tx 7

If $(\operatorname{Val}($ Text5.Text $)<\operatorname{Val}(\mathrm{NH} 4(1)))$ Then

WQINH4 $=100$

ElseIf $(\operatorname{Val}($ Text5.Text) $>=\operatorname{Val}(\mathrm{NH} 4(5)))$ Then

WQINH4 = 1

Else

For $\mathrm{i}=1$ To 4

If $((\mathrm{Val}($ Text5.Text) $>=\operatorname{Val}(\mathrm{NH} 4(\mathrm{i})))$ And

$(\operatorname{Val}($ Text5.Text $)<\operatorname{Val}(\mathrm{NH} 4(\mathrm{i}+1))))$ Then

WQINH4 $=(($ WQ1 $(\mathrm{i})-\mathrm{WQ1}(\mathrm{i}+1)) *$

$(\mathrm{NH} 4(\mathrm{i}+1)-$ Text5.Text) / (NH4(i +1$)-$ NH4(i))) + WQ1(i + 1)

End If

Next

End If

Else

WQINH4 $=0$

End If

If Check6.Value Then

Text6. Text $=$ Tx 8

If $(\operatorname{Val}($ Text6.Text $)<\operatorname{Val}(\mathrm{PO} 4(1)))$ Then

WQIPO4 $=100$

ElseIf $(\operatorname{Val}($ Text6.Text) $>=\operatorname{Val}(\operatorname{PO} 4(5)))$

Then

WQIPO4 = 1

Else

For $\mathrm{i}=1$ To 4

If $((\operatorname{Val}($ Text6.Text) $>=\operatorname{Val}(\mathrm{PO} 4(\mathrm{i})))$ And

$(\operatorname{Val}($ Text6.Text $)<\operatorname{Val}(\mathrm{PO} 4(\mathrm{i}+1))))$ Then

WQIPO4 $=(($ WQ1 $(\mathrm{i})-\mathrm{WQ1}(\mathrm{i}+1)) *$

$(\mathrm{PO} 4(\mathrm{i}+1)$ - Text6. Text) / (PO4(i +1$)-\mathrm{PO} 4(\mathrm{i})))$

+ WQ1 $(i+1)$

End If
Next

End If

Else

WQIPO4 $=0$

End If

If Check7.Value Then

Text7.Text $=\mathrm{Tx} 2$

If $(\operatorname{Val}($ Text7.Text $)<\operatorname{Val}(\operatorname{TSS}(1)))$ Then

WQITSS $=100$

ElseIf $(\operatorname{Val}($ Text7.Text $)>\operatorname{Val}(\operatorname{TSS}(5)))$ Then

WQITSS $=1$

Else

For $\mathrm{i}=1$ To 3

If $((\operatorname{Val}($ Text7.Text $)>=\operatorname{Val}(\operatorname{TSS}(\mathrm{i})))$ And $(\operatorname{Val}($ Text7.Text $)<=\operatorname{Val}(\operatorname{TSS}(\mathrm{i}+1))))$ Then

WQITSS $=(($ WQ1 $(i)-$ WQ1 $(i+1)) *$ $(\operatorname{TSS}(\mathrm{i}+1)-$ Text7.Text $) /(\operatorname{TSS}(\mathrm{i}+1)-\mathrm{TSS}(\mathrm{i})))$ + WQ1 $(i+1)$

End If

Next

End If

Else

WQITSS $=0$

End If

If Check8.Value Then

Text8.Text $=\mathrm{Tx} 3$

If $(\operatorname{Val}($ Text8.Text $)<\operatorname{Val}(\mathrm{NTU}(1)))$ Then

WQINTU $=100$

ElseIf $(\operatorname{Val}($ Text8.Text $)>=\operatorname{Val}(\mathrm{NTU}(5)))$ Then

$\mathrm{WQINTU}=1$

Else

For $\mathrm{i}=1$ To 4

If $((\operatorname{Val}($ Text8.Text $)>=\operatorname{Val}(\mathrm{NTU}(\mathrm{i})))$ And $(\operatorname{Val}($ Text8.Text $)<\operatorname{Val}(\mathrm{NTU}(\mathrm{i}+1))))$ Then

WQINTU $=(($ WQ1 $(\mathrm{i})-\mathrm{WQ1}(\mathrm{i}+1)) *$ $(\mathrm{NTU}(\mathrm{i}+1)-$ Text8.Text $) /(\mathrm{NTU}(\mathrm{i}+1)-$ NTU(i))) + WQ1(i + 1)

End If

Next

End If

Else

WQINTU $=0$

End If 
If Check9.Value Then

Text9. Text $=$ Tx9

If $(\operatorname{Val}($ Text9.Text $)<\operatorname{Val}(\mathrm{CLF}(1)))$ Then

$\mathrm{WQICLF}=100$

Then

ElseIf $(\operatorname{Val}($ Text9.Text) $>\operatorname{Val}(\operatorname{CLF}(5)))$

$\mathrm{WQICLF}=1$

Else

For $\mathrm{i}=1$ To 3

If $((\operatorname{Val}($ Text9.Text $)>=\operatorname{Val}(\mathrm{CLF}(\mathrm{i})))$ And $(\operatorname{Val}($ Text9.Text $)<=\operatorname{Val}(\mathrm{CLF}(\mathrm{i}+1))))$ Then

WQICLF $=(($ WQ1 $(\mathrm{i})-\mathrm{WQ1}(\mathrm{i}+1)) *(\mathrm{CLF}(\mathrm{i}$

$+1)-$ Text9.Text $) /(\operatorname{CLF}(i+1)-\operatorname{CLF}(\mathrm{i})))+$ WQ1 $(i+1)$

End If

Next

End If

Else

WQICLF $=0$

End If

If Check10.Value Then

Text10.Text $=$ Tx 1

If $(\operatorname{Val}($ Text10.Text $)<=5.5)$ Then

WQIpH = 1

ElseIf $((\operatorname{Val}($ Text10.Text) $>5.5)$ And

$(\operatorname{Val}($ Text10.Text $)<6))$ Then

$\mathrm{WQIpH}=((\mathrm{WQ3}(3)-\mathrm{WQ3}(2)) *(\mathrm{pH}(3)-$

Text10.Text) / (pH(3) - pH(2))) + WQ3(2)

ElseIf $((\operatorname{Val}($ Text10.Text $)>=6)$ And

$(\operatorname{Val}($ Text10.Text $)<=8.5))$ Then

WQIpH $=100$

ElseIf $((\operatorname{Val}($ Text10.Text $)>8.5)$ And

$(\operatorname{Val}($ Text10.Text $)<9))$ Then

$\mathrm{WQIpH}=((\mathrm{WQ3}(4)-\mathrm{WQ3}(5)) *(\mathrm{pH}(5)-$

Text10.Text) / (pH(5) - pH(4)))+WQ3(5)

Else

WQIpH $=1$

End If

Else

WQIpH $=0$

End If

WQI1 $=0$

$\operatorname{dem}=0$

If (WQIDO $<>0$ ) Then

WQI1 = WQI1 + WQIDO $\operatorname{dem}=\operatorname{dem}+1$

Else

$$
\text { WQI1 = WQI1 }
$$

End If

If (WQIBOD $<>0$ ) Then

WQI1 = WQI1 + WQIBOD

$\operatorname{dem}=\operatorname{dem}+1$

Else

WQI1 = WQI1

End If

If (WQICOD $<>0$ ) Then

WQI1 = WQI1 + WQICOD

$\operatorname{dem}=\operatorname{dem}+1$

Else

$$
\text { WQI1 = WQI1 }
$$

End If

If (WQINH4 $<>0$ ) Then

WQI1 = WQI1 + WQINH4

dem $=$ dem +1

Else

$$
\text { WQI = WQI }
$$

End If

If (WQIPO4 $<>0$ ) Then

$$
\text { WQI1 = WQI1 + WQIPO4 }
$$

$\operatorname{dem}=\operatorname{dem}+1$

Else

WQI1 = WQI1

End If

If dem $<>0$ Then

WQI1 = (WQI1 / dem $)$

Else

$\mathrm{WQI1}=0$

End If

$\mathrm{WQI} 2=0$

dem $=0$

If (WQITSS $<>0$ ) Then

$\mathrm{WQI} 2=\mathrm{WQI} 2+\mathrm{WQITSS}$

$\mathrm{dem}=\operatorname{dem}+1$

Else

WQI2 = WQI2

End If

If (WQINTU $<>0$ ) Then

$\mathrm{WQI}=\mathrm{WQI} 2+\mathrm{WQINTU}$

$\operatorname{dem}=\operatorname{dem}+1$

Else 


\section{BÀI BÁO KHOA HỌC}

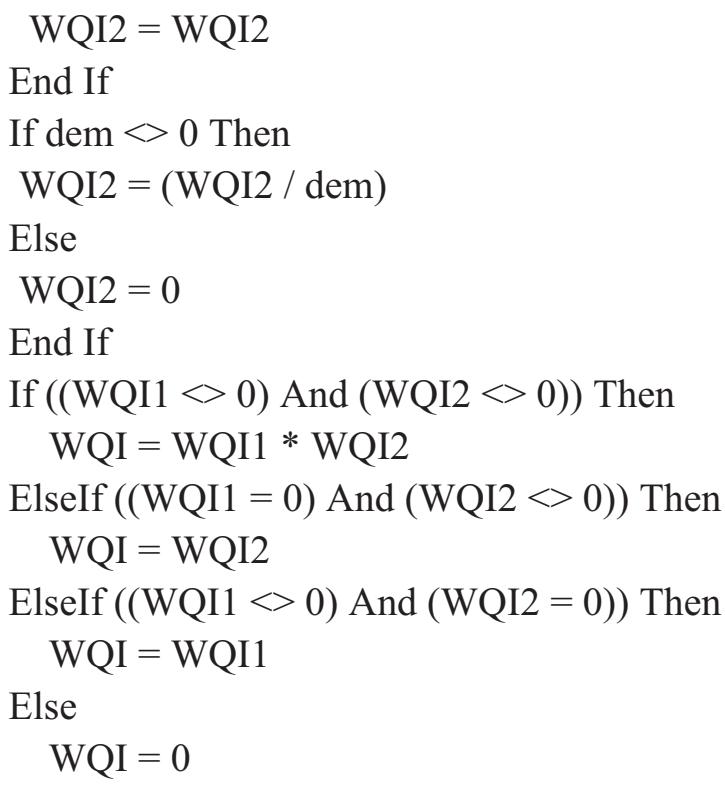

End If

If ((WQICLF $<>0)$ And (WQI $<>0)$ ) Then

$\mathrm{WQI}=\mathrm{WQI} *$ WQICLF

ElseIf $((\mathrm{WQICLF}=0)$ And $(\mathrm{WQI}<>0))$ Then

$\mathrm{WQI}=\mathrm{WQI}$ Then

ElseIf ((WQICLF $<>0)$ And $(\mathrm{WQI}=0))$

$\mathrm{WQI}=\mathrm{WQICLF}$
Else

$$
\mathrm{WQI}=0
$$

End If

$\mathrm{WQI}=\mathrm{WQI}^{\wedge}(1 / 3)$

If ((WQIpH $<>$ ) And (WQI $<>0)$ ) Then

$\mathrm{WQI}=(\mathrm{WQI} * \mathrm{WQIpH}) / 100$

ElseIf $((\mathrm{WQIpH}<>0)$ And $(\mathrm{WQI}=0))$ Then

WQI $=$ WQIpH

ElseIf $((\mathrm{WQIpH}=0)$ And $(\mathrm{WQI}<>0))$ Then

$$
\mathrm{WQI}=\mathrm{WQI}
$$

Else

$\mathrm{WQI}=0$

End If

Open Out For Output As 2

Print \#2, WQI

Close (2)

End Sub

\subsection{Các bước sử dụng}

Bước 1:

Vào thư mục "Software WQI", mở file "CLN.txt" để nhập các chỉ số tính toán WQI. Các chỉ số lần lượt là: $\mathrm{pH}, \mathrm{TSS}, \mathrm{DODUC}, \mathrm{DO}$, $\mathrm{BOD}_{5}, \mathrm{COD}, \mathrm{NH}_{4+}, \mathrm{PO}_{4}{ }^{3-}$, Coliforms, $\mathrm{T}^{\circ} \mathrm{C}$ như hình dưới đây:

\begin{tabular}{l}
\hline CLN.txt - Notepad \\
File \\
\hline 7.1
\end{tabular}

Hình 3. Dũ liệu tính toán chất lương nước WQI

Bước 2:

Vào thư mục "Software WQI", mở file "WQI_Srepok.exe" để chạy phần mềm

Bước 3:

Khi phần mềm hiển thị lên với giao diện như Hình 1:

- Tích vào vị trí có mẫu quan trắc chất lượng nước bên khối "Bản đồ lưu vực sông"

- Tích vào các thông số chất lượng nước bên khối "Chọn chỉ tiêu"

- Click vào nút "Tinh toan" để tính chỉ số WQI (Hình 3)

- Click vào nút "Hiển thi" để hiển thị kết quả chất lượng nước (Hình 4).

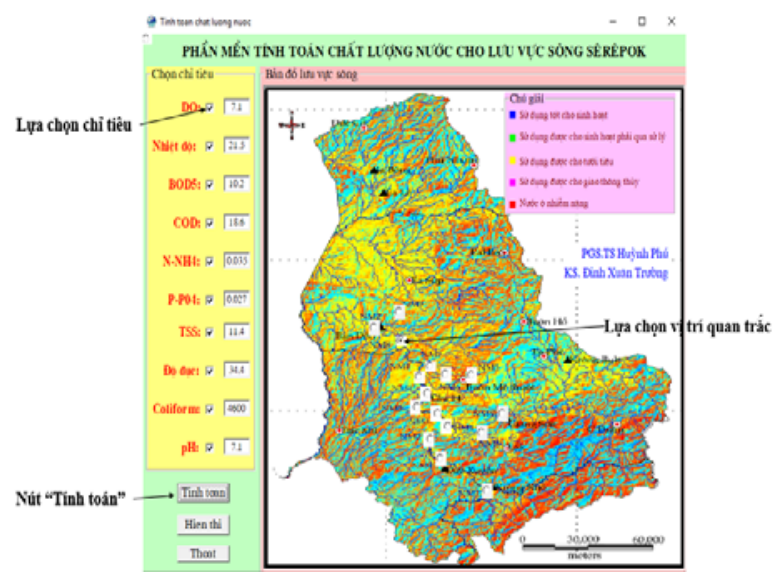

Hình 4. Tính toán chỉ số WQI 


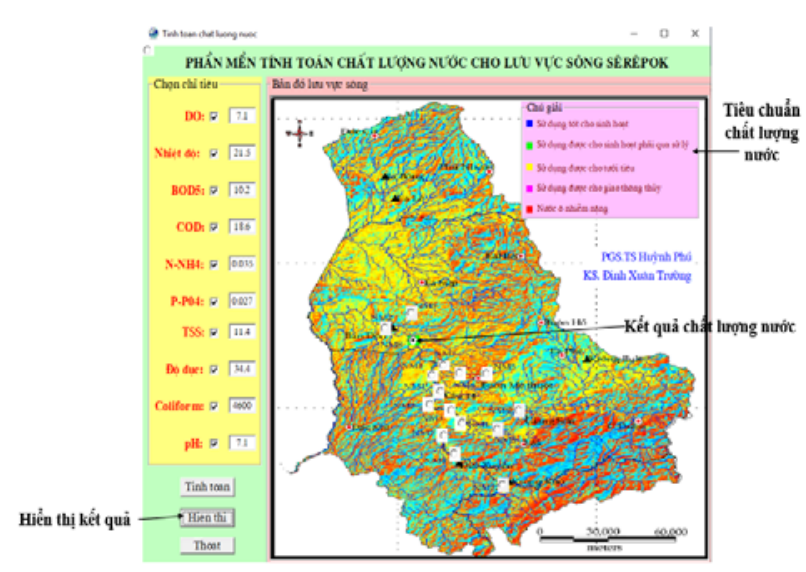

Hình 5. Chất lượng nước tại vị trí quan trắc, lấy mẫu

Ghi chú: Nếu muốn tính chất lượng nước cho các vị trí khác, thực hiện lại các bước tù Bước 1 đến Bước 3.

\section{Kết luận và kiến nghị}

Lưu vực sông Serepok có vai trò quan trọng trong phát triển Kinh tế xã hội, an ninh quốc phòng, quan hệ quốc tế và bảo vệ môi trường tại Tây nguyên. Lưu vực sông hiện nay đã và đang khai thác tài nguyên nước mặt phục vụ nông lâm nghiệp, thủy điện... Tuy nhiên TNN phân bố không đồng đều theo không gian và thời gian. Việc tính toán WQI theo hướng dẫn của Bộ tài nguyên môi trường đã đem lại những thuận lợi cho việc đánh giá chất lượng nước. Tuy nhiên, để cung cấp nhanh chóng thông tin về chất lượng nước nhằm đưa ra các giải pháp nhanh chóng mà không phải tính toán thống kê, bằng phương pháp xây dựng phần mềm tin học WQI_Serepok giúp cho việc tính toán nhanh, xác định ngay các vị trí đang bị ô nhiễm trên sông bằng những thao tác đơn giản nhanh chóng, không cần nhiều số liệu quan trắc khi nhập vào phần mềm sẽ nhận thấy những vị trí ô nhiễm, mức độ ô nhiễm. Từ đó đưa ra những quyết định nhanh chóng kịp thời trong công tác quản lý chất lượng nguồn nước của lưu vực sông.

\section{Tài liệu tham khảo}

1. Báo cáo NCKH cấp Bộ (2006), Nghiên cưu cơ sở khoa học và giải pháp công nghệ để phát triển bền vũng lưu vục sông Hồng. Viện Quy hoạch Thủy lợi.

2. Báo cáo NCKH cấp Bộ (2004), Nghiên cưu cơ sở khoa học và kinh nghiệm thực tiến Quản lý tổng hợp tài nguyên nước lưu vực sông $\mathrm{Ba}$. Đại học Thủy Lợi.

3. Bộ Tài nguyên và môi trường (2011), Sổ tay hướng dẫn tính toán chất lượng nước, Hà Nội.

4. Chi cục thống kê Đắk Lắk (2017), Niên giám thống kê tỉnh Đắk Lắk.

5. Chính Phủ (2008), Nghị định 112/2008/NĐ-CP về về quản lý, bảo vệ, khai thác tổng hơp tài nguyên và môi trường các hồ chứa thủy điện, thủy lợi.

6. Nguyễn Văn Hạnh (2010), Đề tài Nghiên cứu xác định dòng chảy môi truò̀ng của hệ thống sông Hồng - sông Thái Bình và đề xuất các giải pháp duy trì dòng chảy môi truờng phù hợp với các yêu cầu phát triển bền vũng tài nguyên nước, Viện Khoa học Thủy lợi Việt Nam.

7. Huỳnh Phú (2013), Nghiên cưu xây dụng bộ số liệu cho việc ứng dụng mô hình toán mô phỏng diễn biến chất lượng nước sông La Ngà Bình Thuận. Tạp chí Khí tượng Thủy văn, 632, tr. 26-32.

8. Huỳnh Phú (2015), Mô hình toán thủy văn môi trương nghiên cưu chất lượng nước vùng ven biển Trà Vinh. Hội thảo: Nghiên cứu khoa học gắn kết với đào tạo Đại học và Sau đại học tại Trường Đại học Tài nguyên và môi trường Hà nội. ISBN. NXB Lao động, tr 184 - 192.

9. Huỳnh Phú (2018), Tác động của công trình hồ đập tới dòng chảy hạ lưu sông La ngà, Úng dụng mô hình thủy văn thưy lực phục hồi dòng chảy tụ nhiên sau khi có hồ chứa Hàm thuận - Đa $m i$. Tạp chí Khí tượng Thủy văn, 686, tr. 01- 11. 


\title{
BÀI BÁO KHOA HỌC
}

\section{RESEARCH ON THE APPLICATION OF WATER QUALITY WQI SOFTWARE TO SEREPOK RIVER BASIN}

\author{
Huynh Phu ${ }^{1}$ \\ ${ }^{1}$ Ho Chi Minh City University of Technology
}

\begin{abstract}
Water Quality Index (WQI) is an index calculated from water quality monitoring parameters, WQI is used to assess and quantify the water quality of water source; WQI has been employed on a scale in many areas since 2011; According to Decision 879/QD-TCMT, July 1, 2011 of Vietnam Environment Administration. Currently, many countries have developed and applied the WQI. Through a calculation model, from different parameters $\mathrm{pH}, \mathrm{DO}, \mathrm{BOD}, \mathrm{COD}, \mathrm{NH}_{4}^{+}, \mathrm{PO}_{4}{ }^{2-}$, Coliform ... the index of water quality will be produced. Water quality can then be compared with each other through the WQI. This paper presents, by informatic tool, WQI_Serepok software to calculate water quality index, contributing to the management of water quality of Serepok River.
\end{abstract}

Keywords: Water quality, WQI, Load bearing, Serepok River, WQI_Serepok software. 